\title{
Risk Factors for Chemotherapy-Induced Leukopenia in Patients with Lung Cancer
}

\author{
Hongyan Jin'1, Xiaoya Chen², Wei He' ${ }^{1}$, Qian Liu' ${ }^{1}$, Zexiong Wei ${ }^{1}$, Jinjun Li2* $^{*}$ \\ ${ }^{1}$ Department of Oncology, Puren Hospital Affiliated to Wuhan University of Science and Technology, \\ Wuhan, China \\ ${ }^{2}$ Medical College of Wuhan University of Science and Technology, Wuhan, China \\ Email:"entry2003@126.com
}

Received 4 February 2016; accepted 15 March 2016; published 18 March 2016

Copyright (C 2016 by authors and Scientific Research Publishing Inc.

This work is licensed under the Creative Commons Attribution International License (CC BY).

http://creativecommons.org/licenses/by/4.0/

(c) (i) Open Access

\begin{abstract}
Objective: This analysis was conducted to clarify risk factors for chemotherapy-induced leukopenia (CIL) in lung cancer. Methods: A retrospective study was conducted on data from 358 patients with lung cancer who received chemotherapy. Results: Among 358 cases of lung cancer who received chemotherapy, a total of 240 patients experienced CIL, rate was $67 \%$. The demographic data including gender $(P=0.795)$, age $(P=0.134)$, presence of selected chronic comorbidities $(P=$ 0.23) were not significantly different in the two groups. The weight loss rate, PS score, sub-normal pre-WBC level, sub-normal pre-PLT level, and the cycle of chemotherapy were significantly different between the groups $(\mathrm{P}<0.05)$. Multivariate analysis revealed that the weight loss rate $\geq 5 \%$ $(O R=0.503)$, sub-normal pre-WBC level $(O R=11.807)$, the cycle of chemotherapy $\geq 3(O R=3.100)$ were main risk factors for CIL in lung cancer. Conclusion: Before treatment, weight loss rate is $5 \%$ or higher, chemotherapy has a cycle of 3 or more and sub-normal WBC level is independent risk factor of lung cancer after chemotherapy-induced leucopenia.
\end{abstract}

\section{Keywords}

Chemotherapy-Induced Leucopenia, Risk Factors, Lung Cancer

\section{Introduction}

This template, created in MS Word 2007, provides authors with most of the formatting specifications needed for preparing electronic versions of their papers. All standard paper components have been specified for three reasons: 1) ease of use when formatting individual papers, 2) automatic compliance to electronic requirements that facilitate the concurrent or later production of electronic products, and 3) conformity of style throughout a jour-

"Corresponding author.

How to cite this paper: Jin, H.Y., Chen, X.Y., He, W., Liu, Q., Wei, Z.X. and Li, J.J. (2016) Risk Factors for Chemotherapy-Induced Leukopenia in Patients with Lung Cancer. International Journal of Clinical Medicine, 7, 225-230. 
nal paper. Margins, column widths, line spacing, and type styles are built-in; examples of the type styles are provided throughout this document and are identified in italic type, within parentheses, following the example. Some components, such as multi-leveled equations, graphics, and tables are not prescribed, although the various table text styles are provided. The formatter will need to create these components, incorporating the applicable criteria that following. Lung cancer continues to be one of the most severe global health problems, and the leading cause of cancer deaths worldwide [1]. The main treatment for Lung cancer includes chemotherapeutic drugs that have various side effects, of which leukopenia is the most common [2]. Chemotherapy-induced leucopenia (CIL) has been reported to be associated with substantial morbidity, mortality, and healthcare costs, suggesting that CIL may be a biological measure of drug activities and disease control [3] [4]. The response of leukemic cells to chemotherapy depends on the level of active drugs reaching the target and the sensitivity to these drugs. These factors also have effect on the response of non-malignant hematopoietic cells. In this study, data from 358 lung cancer patients are reviewed to investigate the CIL potential high-risk factors and the precaution measures to reduce its occurrence.

\section{Patients and Methods}

\subsection{Inclusion Criteria}

Eligible patients were adults (aged 20 to 89 years) with a histologically confirmed lung cancer. Routine blood examination was conducted before and after every chemotherapy cycle. We included the lowest white cell count in the study, if patients receiving several chemotherapy cycles. In addition, data field grouping involved: patients demographics, clinical details, treatment history and outcome data.

\subsection{Exclusion Criteria}

1) Patients combined with severe heart, liver, kidney or blood system diseases.

2) Patients received granulocyte colony-stimulating factor (G-CFS) or other leukogenic drugs during the course of treatments.

\subsection{Patients Characteristic}

Patients characteristic concluded: age, gender, presence of selected chronic comorbidities (cardiovascular disease, diabetes, liver disease, renal disease), the weight loss rate, PS score (ECOG/WHO performance status), liver and renal function (routine blood test including ALT, AST, Urea, Creatinine), routine blood counts (routine blood test including pre-WBC, pre-PLT, pre-RBC), stages of lung cancer, bone metastasis, relative dose intensity, the cycle number and radiation therapy. Only observed data were utilized in defining patient characteristics.

\subsection{Statistical Analysis}

A Chi-square test assessed the association between the categorical variables and the degree of leukopenia. Then descriptive analyses were used to construct the population profile and a variety of bivariate tests were accomplished to probe potential relationships between the independent pools of socio-demographic and clinical variables and chemotherapy-induced leucopenia in the population.

The results are presented as $\mathrm{P}$ value, odds ratios and 95\% confidence intervals for variables in the chemotherapy-induced leucopenia prediction model, to provide information about their effect measure. The Wald test was used to determine the variables in the final model. The P values were two-sided and all the probability criteria for the variables were 0.05. All statistical operations were performed using the SPSS statistical software 17 .

\section{Results}

A total of 358 patients diagnosed with lung cancer were divided into CIL group and non-CIL group (matched group), according to the leucocyte count. There were 240 patients in CIL group, 74.2\% were man, 25.8\% were woman. Among them, 128 patients were at the advanced age (60 - 89 years old), 112 young patients (20 - 59 years old) and $39.6 \%$ patients had some chronic comorbidities. On the other hand, 118 patients were in the matched group, 72.9\% were man, 27.1\% were woman. Among them, 53 patients were at the advanced age (60 89 years old), 65 young patients (20 - 59 years old) and 33.1\% patients had some chronic comorbidities. The 
demographic data concluding gender $(\mathrm{P}=0.795)$, age $(\mathrm{P}=0.134)$, presence of selected chronic comorbidities ( $\mathrm{P}$ $=0.23$ ) were not significantly different in the two groups. We included 13 potential risk factors into analysis, among which the weight loss rate $(P=0.008)$, PS score $(P=0.008)$, sub-normal pre-WBC level $(P=0.003)$, sub-normal pre-PLT level $(\mathrm{P}=0.02)$ and the cycle of chemotherapy $(\mathrm{P}=0.001)$ were significantly different between the groups. (Table 1, Table 2).

A bivariate test was performed to further analyze these 5 risk factors, and the final selection of the 3 factors were statistically significant. So, the weight loss rate $\geq 5 \%$ ( $O R=0.503, P=0.008)$, sub-normal pre-WBC level $(\mathrm{OR}=11.807, \mathrm{P}=0.003)$, the cycle of chemotherapy $\geq 3(\mathrm{OR}=3.100, \mathrm{P}=0.001)$ were main risk factors (Table 3, Table 4).

\section{Discussion}

Lung cancer is a malignant lung tumor characterized by uncontrolled cell growth in tissues of the lung, which is often caused by a combination of genetic factors and exposure to asbestos, or other forms of air pollution, including second-hand smoke. The main clincical types include small-cell lung carcinoma (SCLC) and non-smallcell lung carcinoma (NSCLC), and the diagnosis is confirmed by biopsy which is usually performed by bronchoscopy or CT-guidance. Chemotherapy is the main treatment, which improves survival and is used as firstline treatment especially in advanced lung carcinoma.

Chemotherapy-induced leucopenia is one of the major dose-limiting toxicities seen in clinical oncology practice. Leucopenia is clinically relevant issues with a negative impact on quality of life, Lead to increased morbidity and mortality, increased treatment costs [5]-[7]. Recent studies have indicated that the occurrence rate of CIL (white blood cell count $<2 \times 109 / \mathrm{L}$ ) was $16 \%$ to $59 \%$ [8]. So, the risk assessment of CIL in patients with lung cancer chemotherapy has important clinical significance.

This retrospective study analyzed clinical data of 358 lung cancer patients, revealing the weight loss rate $\geq 5 \%$, sub-normal pre-WBC level, the cycle of chemotherapy $\geq 3$ were the main risk factors.

The reduced probability of various hematopoietic cells in bone marrow is determined by the length of the life of the cell. In our model, a higher baseline leucocyte count appears to be protective against chemotherapy-induced leukopenia. Before treatment, the low level of white blood cells reflect bone marrow hematopoietic function has a certain degree of reduction, which is more prone to bone marrow suppression after chemotherapy. In the

Table 1. Risk factors for leukopenia in lung cancer patients receiving chemotherapy.

\begin{tabular}{|c|c|c|c|c|c|}
\hline & & CIL & No CIL & & \\
\hline & & $n=240$ & $n=118$ & $X^{2}$ & $P$ value \\
\hline \multirow{2}{*}{ Gender } & Male & 178 & 86 & \multirow{2}{*}{0.252} & \multirow{2}{*}{0.795} \\
\hline & Female & 62 & 32 & & \\
\hline \multirow{2}{*}{ Age } & $20-59 y$ & 112 & 65 & \multirow{2}{*}{2.243} & \multirow{2}{*}{0.134} \\
\hline & $60-89 y$ & 128 & 53 & & \\
\hline \multirow[b]{2}{*}{$\begin{array}{c}\text { Presence of selected chronic } \\
\text { comorbidities }\end{array}$} & No & 145 & 79 & & \\
\hline & Yes & 95 & 39 & 1.441 & 0.23 \\
\hline \multirow[b]{2}{*}{ Weight loss rate } & $\leq 5 \%$ & 198 & 83 & \multirow{2}{*}{6.93} & \multirow[b]{2}{*}{0.008} \\
\hline & $\geq 5 \%$ & 42 & 35 & & \\
\hline \multirow[t]{4}{*}{ PS score } & & & & \multirow[t]{4}{*}{9.707} & \multirow[t]{2}{*}{0.008} \\
\hline & 0 & 74 & 56 & & \\
\hline & 1 & 137 & 53 & & \\
\hline & 2 & 29 & 9 & & \\
\hline \multirow{2}{*}{ Gender } & Male & 178 & 86 & \multirow{2}{*}{0.252} & \multirow{2}{*}{0.795} \\
\hline & Female & 62 & 32 & & \\
\hline
\end{tabular}


Table 2. Risk factors for leukopenia in lung cancer patients receiving chemotherapy.

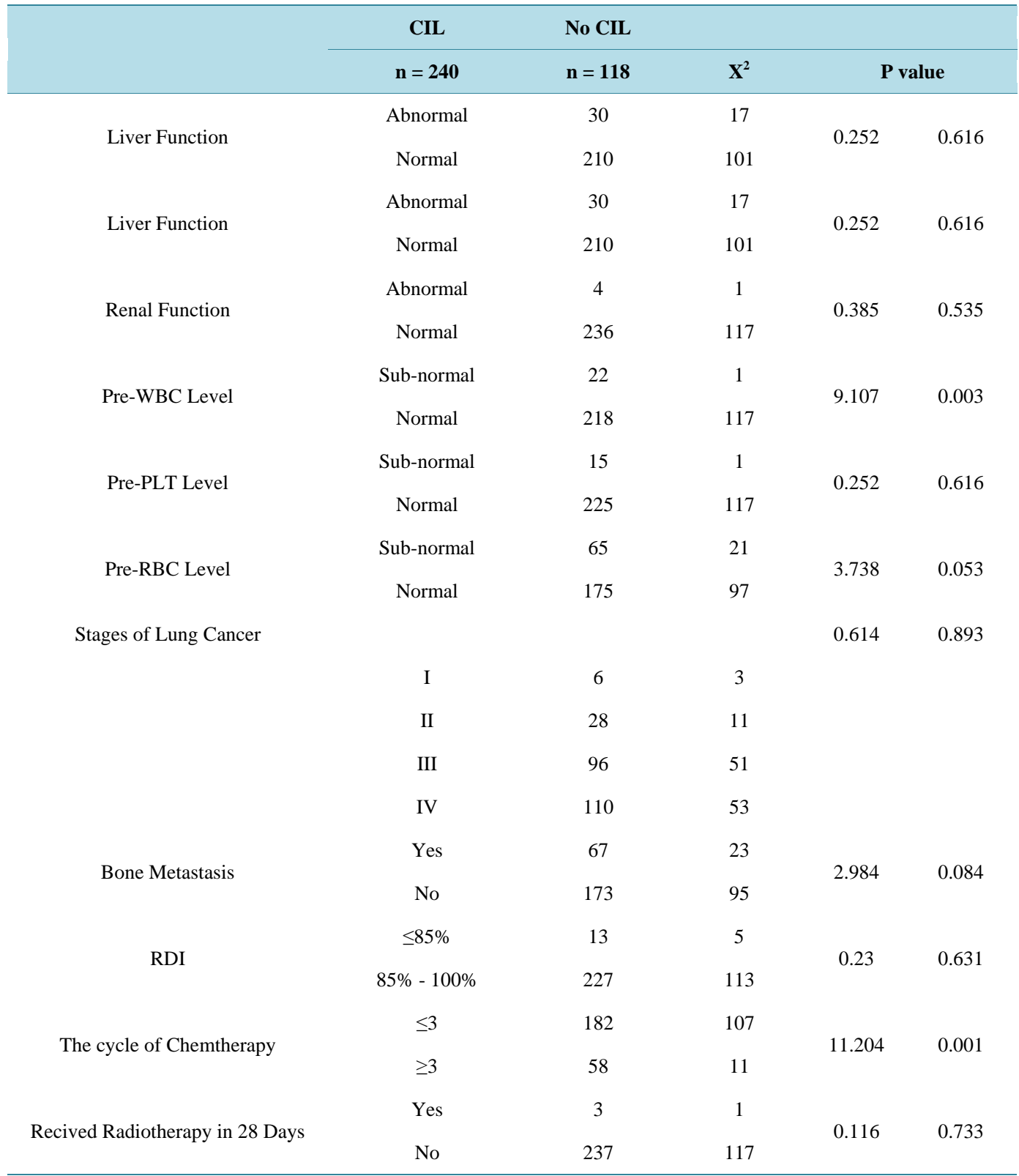

TIPS: (a) ALT > 40 IU/L; (b) $\mathrm{Cr}>88.4 \mu \mathrm{mol} / \mathrm{L}$; ${ }^{*} \mathrm{RDI}=$ Relative dose intensity.

Table 3. Logistic binary regression analysis variable assignment.

\begin{tabular}{|c|c|c|c|}
\hline & Variable & Assignment & \\
\hline & $\mathrm{Y}$ & $0=$ No CIL, $1=\mathrm{CIL}$ & \\
\hline & Waight loss rate & $0 \% \leq 5 \%, 1 \% \geq 5 \%$ & \\
\hline & PS score & $1=0,1=1,2=2$ & \\
\hline Sub-normal & Pre-WBC level & $0=$ normal, & $1=$ reduce \\
\hline Sub-normal & Pre-PLT level & 0 = normal, & 1 = reduce \\
\hline The cycle of & Chemotherapy & $0 \leq 3$ & $1 \geq 3$ \\
\hline
\end{tabular}


Table 4. Logistic binary regression model predicting CIL in patients with lung cancer initiating chemotherapy.

\begin{tabular}{|c|c|c|c|}
\hline & Variable & Assignment & \\
\hline & $\mathrm{Y}$ & $0=$ No CIL, $1=\mathrm{CIL}$ & \\
\hline & Waight loss rate & $0 \% \leq 5 \%, 1 \% \geq 5 \%$ & \\
\hline & PS score & $1=0,1=1,2=2$ & \\
\hline Sub-normal & Pre-WBC level & $0=$ normal & $1=$ reduce \\
\hline Sub-normal & Pre-PLT level & $0=$ normal, & $1=$ reduce \\
\hline The cycle of & Chemotherapy & $0 \leq 3$ & $1 \geq 3$ \\
\hline
\end{tabular}

study, the patients of low white blood cells before treatment are 22 (9.2\%) in CIL group, and 1 (0.85\%) in matched group $(\mathrm{P}=0.003<0.05)$. Multivariate analysis indicated that the possibility of CIL in patients with low level of white blood cells was 11.807 times that of matched group $(\mathrm{P}=0.036<0.05)$, showing the important value of the detection index in predicting the occurrence of CIL. Combined with the present study, it is suggested that the occurrence of white blood cell reduction may be related to the following reasons: 1) In the late stage of tumor, tumor cells have been spread to the myeloid hematopoietic system, which directly inhibition the bone marrow hematopoietic function [9]. 22 cases of patients with a leucocytosis before treatment, in CIL group, included 7 cases with bone metastasis. 2) The patients had received multiple chemotherapy, who is at the stage of bone marrow suppression. 3) Most patients have gastrointestinal adverse reactions after chemotherapy, resulting in cacotrophy, thereby affecting the bone marrow hematopoietic.

The greater the tumor load in patients with advanced lung cancer, the more nutrients the cancer cells consume. Moreover, tumor cells secrete the so called "anorexia factors", also can lead to the loss of appetite. After chemotherapy, a large number of tumor cells break down the metabolism and increase the burden of the digestive system, appearing different degrees of gastrointestinal reaction, which directly affect the patient's eating and digestion and absorption, resulting in patients with malnutrition, weight decline, thereby affecting the proliferation of bone marrow [10]. The results of this study show that weight loss is closely related to the occurrence of CIL, weight loss rate is more than or equal to $5 \%$ of patients and compared to less than $5 \%$ of patients, the possibility of CIL increased $2.38(1 / 0.296-1)$ times, $\mathrm{P}=0.01<0.05$. Hence, patients with serious digestive tract reaction after chemotherapy should be timely replenished essential nutrients, given antiemetic, acid suppression, and other symptomatic treatments to protect the gastric mucosa and increase appetite, in order to reduce gastrointestinal toxicities of chemotherapy, trying to reduce and avoid the occurrence of severe bone marrow suppression.

The cycle number of the current chemotherapy is a important factor of the current model, and our results indicate that more than or equal to 3 cycles of chemotherapy have a greater risk of chemotherapy-induced leukopenia. Multiple factor analysis indicated that patients who had received 3 or more cycles of chemotherapy patients with CIL were 3.100 times as much as the patients who had received less than 3 cycles of chemotherapy, $\mathrm{P}=0.004<0.05$. The increased risk of CIL after 3 cycles might be related to the contact toxicity and toxic accumulation of chemotherapy drugs. And chemotherapy-induced bone marrow nerve injury impairs hematopoietic regeneration [11], demonstrating that chemotherapy-induced nerve injury in the bone marrow is a critical lesion impairing hematopoietic regeneration. Therefore, we suggest that the patients who had received chemotherapy more than 3 times should be regarded as a high risk group of CIL, closely monitoring the blood routine. It is necessary and feasible to take effective preventive measures to prevent further inhibition of bone marrow function and promote the recovery of bone marrow function.

The occurrence of CIL is influenced by many factors. Clinicians can evaluate the risk of chemotherapy-induced leucopenia in patients with lung cancer to lessen the risk, such as by decreasing the chemotherapy drug dosages and/or starting CSF, reducing the degree of bone marrow suppression after chemotherapy and duration, which would help patients get through high risk of infection safely, implement chemotherapy smoothly and improve the quality of life [12].

\section{Conclusion}

The main risk factors for CIL in lung cancer include 5\% or higher weight loss rate, 3 or more cycles of chemo- 
therapy and sub-normal WBC level. This research may be able to develop a predictive model that clinicians can accurately assess a patient's risk for the development of leukopenic complications during the course of chemotherapy, so that appropriate prophylactic measures can be implemented before the first cycle of chemotherapy in those patients who are at high risks.

\section{References}

[1] Ridge, C.A., McErlean, A.M. and Ginsberg, M.S. (2013) Epidemiology of Lung Cancer. Seminars in Interventional Radiology, 30, 93-98. http://dx.doi.org/10.1055/s-0033-1342949

[2] Liu, W., Zhang, C.C. and Li, K. (2013) Prognostic Value of Chemotherapy-Induced Leukopenia in Small-Cell Lung Cancer. Cancer Biology \& Medicine, 10, 92-98.

[3] Shitara, K., Matsuo, K., Oze, I., et al. (2011) Meta-Analysis of Neutropenia or Leukopenia as a Prognostic Factor in Patients with Malignant Disease Undergoing Chemotherapy. Cancer Chemotherapy and Pharmacology, 68, 301-307. http://dx.doi.org/10.1007/s00280-010-1487-6

[4] Han, Y.F., Gong, Z., Huang, L.Q., Xia, X. and Zhao, W.J. (2010) Clinical Study on Acupuncture for Leukopenia induced by Chemotherapy. Zhongguo Zhen Jiu, 30, 802-805.

[5] Campos, M.I., Vieira, W.D., Campos, C.N., Aarestrup, F.M. and Aarestrup, B.J. (2015) Atorvastatin and Trans-Caryophyllene for the Prevention of Leukopenia in an Experimental Chemotherapy Model in Wistar Rats. Molecular and Clinical Oncology, 3, 825-828. http://dx.doi.org/10.3892/mco.2015.544

[6] Choi, T.Y., Lee, M.S. and Ernst, E. (2015) Moxibustion for the Treatment of Chemotherapy-Induced Leukopenia: A Systematic Review of Randomized Clinical Trials. Support Care Cancer, 23, 1819-1826. http://dx.doi.org/10.1007/s00520-014-2530-7

[7] Karaca, H., Bozkurt, O., Ozaslan, E., et al. (2014) Positive Effects of Oral Beta-Glucan on Mucositis and Leukopenia in Colorectal Cancer Patients Receiving Adjuvant FOLFOX-4 Combination Chemotherapy. Asian Pacific Journal of Cancer Prevention, 15, 3641-3644. http://dx.doi.org/10.7314/APJCP.2014.15.8.3641

[8] Li, J., Dai, C.H., Shi, S.B., Bao, Q.L., Yu, L.C. and Wu, J.R. (2010) Induction Concurrent Chemoradiotherapy Compared with Induction Radiotherapy for Superior Sulcus Non-Small Cell Lung Cancer: A Retrospective Study. AsiaPacific Journal of Clinical Oncology, 6, 57-65. http://dx.doi.org/10.1111/j.1743-7563.2009.01265.x

[9] Khafagy, R., Shackley, D., Samuel, J., O’Flynn, K., Betts, C. and Clarke, N. (2007) Complications Arising in the Final Year of Life in Men Dying from Advanced Prostate Cancer. Journal of Palliative Medicine, 10, 705-711. http://dx.doi.org/10.1089/jpm.2006.0185

[10] Su, Z., Mao, Y.P., OuYang, P.Y., Tang, J., Lan, X.W. and Xie, F.Y. (2015) Leucopenia and Treatment Efficacy in Advanced Nasopharyngeal Carcinoma. BMC Cancer, 15, 429. http://dx.doi.org/10.1186/s12885-015-1442-3

[11] Tendas, A., Niscola, P., Perrotti, A., Dentamaro, T., de Fabritiis, P. and Arcese, W. (2015) Chemotherapy Induced Nausea and Vomiting in Bone Marrow Transplant: The Unmet Need. Support Care Cancer, 23, 2211. http://dx.doi.org/10.1007/s00520-015-2711-z

[12] Pais, I., Correia, N., Pimentel, I., et al. (2014) Effects of Acupuncture on Leucopenia, Neutropenia, NK, and B Cells in Cancer Patients: A Randomized Pilot Study. Evidence-Based Complementary and Alternative Medicine, 2014, Article ID: 217397. http://dx.doi.org/10.1155/2014/217397 